\title{
Distributed Deep Reinforcement Learning Resource Allocation Scheme For Industry 4.0 Device-To-Device Scenarios
}

\author{
Jesús Burgueño(2) Ramoni Adeogun(1) Rasmus Liborius Bruun(1) C. Santiago Morejón García(1) \\ Isabel de-la-Bandera(2) Raquel Barco(2) \\ (1) Department of Electronic Systems, Aalborg University, Denmark \\ (2) Instituto Universitario de Investigación en Telecomunicación (TELMA), \\ Universidad de Málaga, CEI Andalucía TECH, Málaga, Spain \\ E-mail: jesusbr@ic.uma.es, fra, rlb, csmgg@es.aau.dk, ibanderac@ic.uma.es, rbm@ic.uma.es
}

\begin{abstract}
This paper proposes a distributed deep reinforcement learning (DRL) methodology for autonomous mobile robots (AMRs) to manage radio resources in an indoor factory with no network infrastructure. Hence, deep neural networks (DNN) are used to optimize the decision policy of the robots, which will make decisions in a distributed manner without signalling exchange. To speed up the learning phase, a centralized training is adopted in which a single DNN is trained using the experience from all robots. Once completed, the pre-trained DNN is deployed at all robots for distributed selection of resources. The performance of this approach is evaluated and compared to 5G NR sidelink mode 2 via simulations. The results show that the proposed method achieves up to 5\% higher probability of successful reception when the density of robots in the scenario is high.
\end{abstract}

ACKNOWLEDGMENT: This work has been partially funded by Junta de Andalucía (projects EDEL4.0:UMA18FEDERJA-172 and PENTA:PY18-4647) and Universidad de Málaga (I Plan Propio de Investigación, Transferencia y Divulgación Científica). Ramoni Adeogun is supported by the Danish Council for Independent Research, grant no. DFF 9041-00146B. The authors would like to express their profound gratitude to Nokia Standardization Aalborg and Aalborg University for funding the first author's research stay. The authors thank Assoc. Prof. Gilberto Beradinelli for his comments on the manuscript. 